\title{
Web Data Mining Technology and Network Information Security
}

\author{
Liu Saidong \\ Sichuan Vocational and Technical College, Suining, Sichuan, 629000, China
}

Keywords: network management; data mining; data warehouse; online analytical processing

Abstract: This paper discusses the architecture, OLAP technology and data mining technology of Web-based data mining network management system, aiming at the current status and existing problems of network management systems. A network management system based on Web and data mining technology is proposed to provide solutions for the problems existing in network management. The friendship system has positive significance for the improvement of network management status.

\section{Introduction}

With the development of network technology, network resources are increasingly heterogeneous and dynamic. The functions of network management are increasingly complex. Traditional network management technologies cannot meet the needs of large-scale complex network management. It is necessary to introduce data into network management. Mining technology, extracting, transforming and integrating massive data in the network into required data, providing relevant analysis and comprehensive technology, enabling decision makers and analysts to comprehensively and comprehensively analyze data resources in a timely and accurate manner, and extract useful knowledge from them. Efficiently realize integrated management of network information security [1]

\section{Overview of data mining technology}

\subsection{Introduction to the process of data mining}

Data mining is the extraction or "mining" of knowledge from large amounts of data. Data mining is also called knowledge discovery in the database or a synonym for KDD. Data mining is a complete process that mines previously unknown, valid, and usable information from large databases and uses that information to make decisions or enrich knowledge. The general content of each step in the process is as follows:

\subsection{Identify business objects}

Clearly define business problems and recognize that data mining is an important step in data mining. The final structure of the mining is unpredictable, but the problem to be explored should be 
foreseeable. For data mining, data mining is blind and will not succeed ${ }^{[2]}$.

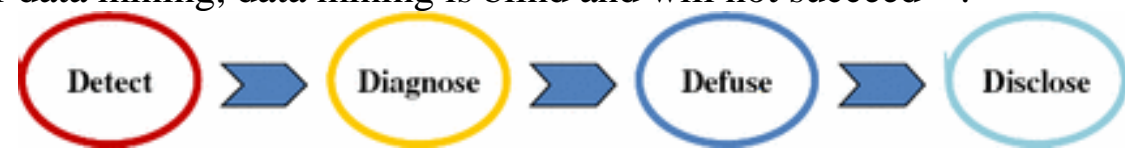

Figure 1 Flow of using Web data mining technology

As shown in Figure 1, we clearly define the business problem. Figure 1 depicts the basic process and main steps of data mining. Network resources are increasingly heterogeneous and dynamic, and the functions of network management are increasingly complex. You should choose an application for data mining to prepare for further analysis.

\subsection{Data Preparation}

The above client only needs to install a web browser, such as IE/NS, so it can be low on the machine itself based on any operating platform that supports Browser. The web server is used to manage the information flow between the client and the application server. Various popular servers, such as IIS, APACHE, etc., can be used by the web server to accept client HTTP requests, and generate dynamic HTML response requests by using static HTML pages or calling the application server. After verification of legality and the like, a request is made to the application server according to the content of the WEB page, and an application such as jsp or asp may be installed on the WEB server, so that information can be exchanged with the application server ${ }^{[1]}$. The application server responds to the request sent by the WEB server, activates the corresponding query task, and utilizes the interface technology to access the data warehouse, such as ODBC and JDBC, and mainly includes the following aspects:

- Selection of data

- Assimilation of knowledge

- Data conversion

- Data mining

- Analysis of results

- Data preprocessing

The choice of data refers to searching all internal and external data information related to business objects, and selecting data suitable for data mining applications; the preprocessing of data refers to the quality of research data, in preparation for further analysis. And determine the type of mining operation to be performed; data conversion refers to the conversion of data into an analysis model, which is built for mining algorithms. Establishing an analytical model that is really suitable for mining algorithms is the key to the success of data mining; data mining refers to mining the resulting transformed data. In addition to perfecting and selecting the appropriate mining algorithm, everything else can be done automatically; the result analysis is done by interpreting and evaluating the results. The analysis method used should generally be based on data mining operations, usually using visualization technology; assimilation of knowledge is the final step, and the knowledge obtained by the analysis is integrated into the organizational structure of the business information system $^{[3]}$.

\section{Application of data mining in network management}

Data mining technology has been application-oriented since its inception. At present, in many fields, data mining is a very fashionable word, especially in the commercial fields such as banking, telecommunications, insurance, transportation, and retail (such as supermarkets). In terms of network management, the TASA system cooperated with a remote communication equipment 
manufacturer in Helsinki University, Finland. The network management alarm database contains a large number of fault feature patterns, which provides a data foundation for data mining. Therefore, it is meaningful to study data mining in network management.

\section{Architecture of network management system based on WEB and data mining}

The WEB-based data warehouse system is a three-layer logical architecture consisting of a presentation layer, an application layer, and a data layer. The architecture is shown in Figure 2. The data layer, the back-end data warehouse, accepts SQL query statements from the application layer and data integration. The WEB server is the function hub of this system, which serves as a bridge between the client and the application server, and can perform data legality check, identity verification, and the like. The workflow of the whole system is as follows: In the presentation layer, the WEB browser user sends a certain operation instruction to the WEB server through the HTrP request submitted by the HTML file; in the application layer, the WEB server responds to the presentation layer with the corresponding application of asp, jsp, etc. Request, and translate into a request acceptable to the application server, or directly translate into a SQL request, the application server completes the corresponding SQL statement and connects with the background data warehouse, issues a SQL query, etc., finally the application layer accepts the results of the data layer, and sends To the client. In addition, the application layer also needs to complete the extraction of data and load it into the data warehouse through data integration. In order to improve efficiency and increase system flexibility, technologies such as java applets, ActiveX, and JavaScript can be introduced to enable the client to have information processing capabilities, thereby reducing the burden on the application layer to some extent ${ }^{[2]}$.

\section{W-D network management system function module}

WD network management system is built on the basis of each existing professional network management. It consists of two functional modules: (1) Management module: It is for each sub-professional network management, mainly processing real-time from various professional network management systems. Information, such as more advanced alarms in the switching network; (2) Analysis and monitoring module: It is divided into different themes for different functional departments, including fault management module (analysis of abnormal conditions of the whole network, alarm monitoring, fault location) Analysis, etc.), performance management module (performance testing, evaluation performance analysis, network performance control, etc.), configuration management module (network status comprehensive analysis, equipment installation monitoring analysis, etc.), security management module (network and network equipment security protection analysis, Intrusion user analysis, billing management module (business billing analysis, business association analysis, etc.). Based on each professional network management, it establishes an analysis model for different topics and levels of each department, and performs data of each sub-professional network management. Integration, using OLAP tools, data mining tools and other knowledge to generate decision reference letters for different topics It also provides supervision functions, strengthens risk prevention and early warning functions, enables network management to achieve the desired goals of decision-making system under the supervision of the supervision system, and realizes the optimal operation of network management on the basis of security. WD network management system The structure is shown in Figure 2: 


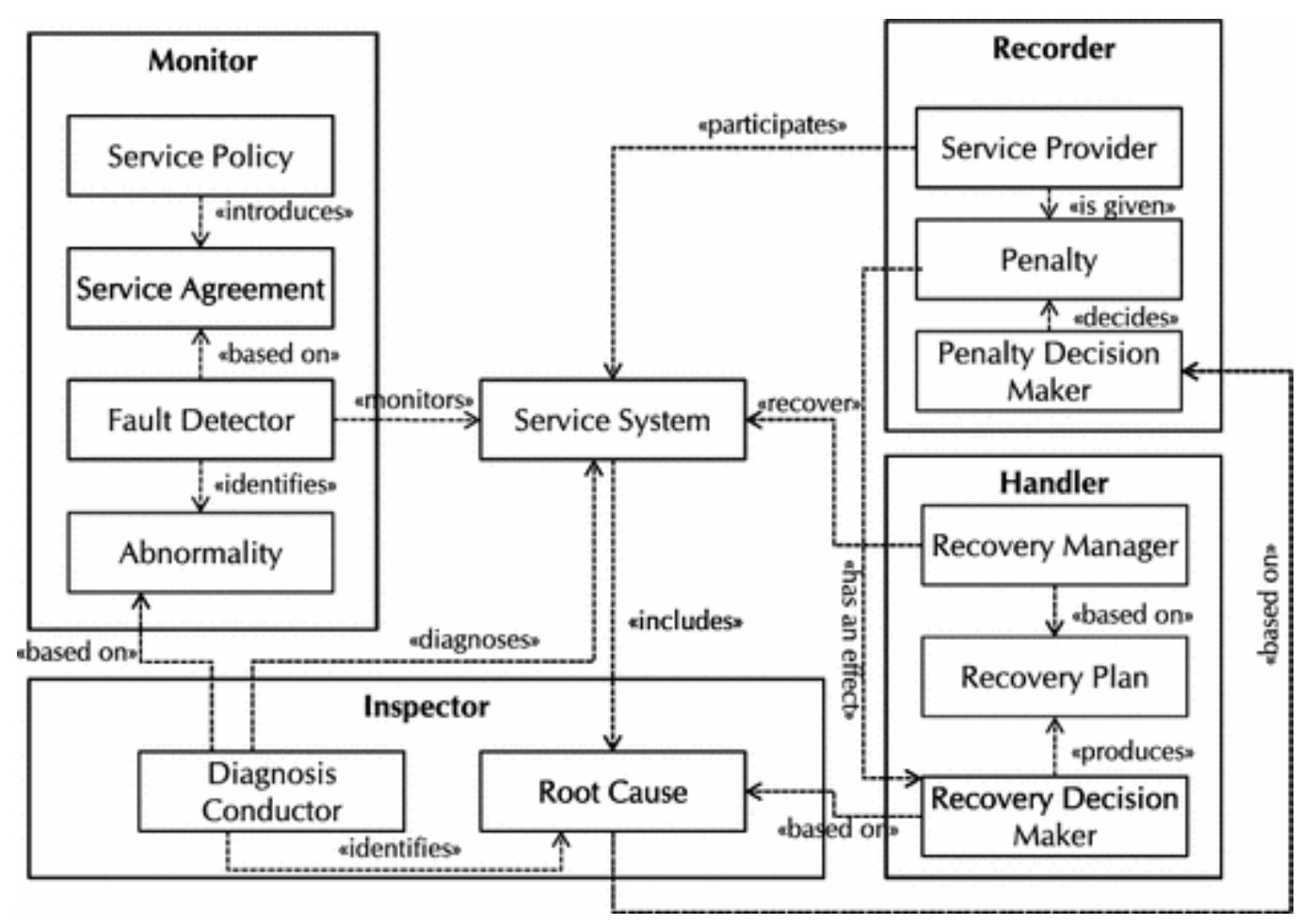

Figure 2 Architecture of the W-D network management system

In Figure 2, the public interface platform can be implemented according to the TMN (Telecom Management Network) and other specifications developed by IT-U, using relevant protocol standards and CORBA and other technologies. The data of each professional network management is extracted, converted and loaded into the data warehouse. Go and form an enterprise-wide global data view. Each department further integrates its own data marts based on its unique theme. The OLAP server further integrates the data information to be analyzed into a multi-dimensional database according to the multi-dimensional analysis model, and further provides services for OLAP and data mining tools. Based on the data in the multi-dimensional database, the OLAP tool performs related data analysis and synthesis on the user's problems. Based on this, the data mining tool automatically discovers potential patterns and new knowledge according to certain algorithms [3].

\section{Conclusions}

The network management system based on Web and data mining technology can not only solve the problem of "information islands" formed by multiple professional network management systems, resources cannot be shared, but also overcome the obstacles of the lack of massive data information in large networks, and introduce data mining technology to a certain extent. Intelligently generate business rules, provide guidance for optimal control networks from a global perspective, help leaders and decision makers prevent risks, improve service and management levels, and provide an effective solution to overcome the shortcomings of current professional network management. It is of practical significance to improve the current status of network management.

\section{References}

[1] Wu L. Effective Integration of Network Information Security and Web Data Mining Technology[J]. Cyberspace Security, 2016.

[2] Wang Y. Based on the Network Information Security and Web Data Mining Technology Integration Research[J]. 
Information Security \& Technology, 2015.

[3] Jiang L, Ren Y. A Network Information Security Prevention Model Base on Web Data Mining[C]// International Conference on Robots \& Intelligent System. IEEE, 2016:26-29. 